\title{
In Vitro Antifungal Activity Against Trychophyton Rubrum of p-Aminochalcones and 3'-Methoxy-4'-Hydroxy Chalcone
}

\author{
Bandeira, P. N.; Fontenele, R. O. S.; Costa, P. S.; Santos, H. S.; Lemos, T. L. G. \\ Rev. Virtual Quim., 2020, 12 (3), 703-711. Data de publicação na Web: 2 de Junho de 2020 \\ http://rvq.sbq.org.br
}

\section{Atividade Antifúngica In Vitro Contra Trychophyton Rubrum de P-Aminochalcones e 3'-Metoxi-4'-Hidroxi Chalcona}

Resumo: O presente estudo relata as atividades antifúngicas avaliadas in vitro de 10 chalconas derivadas da $p$-aminoacetofenona e da 3'-metoxi-4'-hidroxiacetofenona contra quatro estirpes dos dermatófitos Trychophyton rubrum (CEMM 0201 e 0202, LABMIC 0203 e 0204). As p-aminochalonas e 3'-metoxi-4'hidroxi-calconas foram sintetizadas pela reação de condensação do Claisen-Schmidt em meio básico. A CIM foi definida pela menor fração de teste capaz de inibir o crescimento fúngico visualmente detectado. A concentração mínima de fungos (CFM) correspondeu à menor concentração, o que não resultou em crescimento fúngico após 2 dias. As chalconas foram efetivas contra o T. rubrum (CIM de 0.015 to $1.25 \mu \mathrm{g} / \mathrm{mL}$ ).

Palavras-chave: Acetofenona; chalconas; dermatófitos; atividade antifúngica.

\begin{abstract}
This study evaluates the in vitro antifungal activities of 10 chalcones derived from $p$-aminoacetophenone and 3'-methoxy-4'-hydroxyacetophenone against four strains of the dermatophytes of Trychophyton rubrum (CEMM 0201 and 0202, LABMIC 0203 and 0204). The $p$-aminochalcone and 3'-methoxy-4'-hydroxy chalcone derivatives were synthesized via the Claisen-Schmidt condensation reaction conducted under basic conditions. The minimum inhibitory concentration (MIC) was defined as the lowest concentration capable of inhibiting fungal growth. The minimum fungicidal concentration (MFC) corresponds to the lowest concentration that results in no fungal growth after 2 days. The chalcones were effective against T. rubrum (MIC $=0.015-1.25 \mu \mathrm{g} / \mathrm{mL}$ ).
\end{abstract}

Keywords: Acetophenone; chalcones; dermatophytes; anfifungical activity.

\footnotetext{
* Universidade Estadual Vale do Acaraú, Av. da Universidade 850, CEP 62040-370, Sobral-CE, Brasil.

1 bandeirapn@yahoo.com.br DOI: $\underline{10.21577 / 1984-6835.20200056}$
} 
ISSN 1984-6835

\section{In Vitro Antifungal Activity Against Trychophyton Rubrum of p-Aminochalcones and 3'-Methoxy-4'-Hydroxy Chalcone}

\section{Paulo Nogueira Bandeira, ${ }^{\mathrm{a}, *}$ Raquel Oliveira dos Santos Fontenele, ${ }^{\mathrm{a}}$ Patrícia Silva Costa, ${ }^{a}$ Hélcio Silva dos Santos, ${ }^{a}$ Telma Leda Gomes de Lemos ${ }^{b}$}

a Universidade Estadual Vale do Acaru, Centro de Ciências Exatas e Tecnologia, Agrárias e Biológicas Campus da Betânia.

b Universidade Federal do Ceará, Departamento de Química Orgânica e Inorgânica, Campus do Pici, Av. Público, 726, CEP 60020-181, Fortaleza-CE, Brazil.

* bandeirapn@yahoo.com.br

Recebido em 25 de Abril de 2019. Aceito para publicação em 7 de Maio de 2020.

\section{Introduction}

\section{Material and Methods}

2.1. Synthesis and characterization of chalcones

2.2. Fungal strains

2.3. Preparation of inoculum for antifungal sensitivity tests

2.4. Antifungal activity

\section{Results and Discussions}

3.1. Analytical data

3.2. Chemistry

3.3. Antifungal activities

\section{Conclusion}

\section{Introduction}

Chalcones have an open chain flavonoid structure in which the two aromatic rings are joined by a three carbon $\alpha, \beta$-unsaturated carbonyl linker (Figure 1). They can be obtained from natural sources or by synthesis, and are widely distributed in fruits, vegetables, and tea. ${ }^{1,2}$ Their antimicrobial activity and, in particular, antifungal action have been largely attributed to the reactive enone moiety. ${ }^{3-6}$

Dermatophytes are fungi that can cause infections (known as tinea) of the skin, hair, and nails<smiles>O=C(/C=C/c1ccccc1)c1ccccc1</smiles>

Figure 1. Fundamental structure of chalcones 
because of their ability to use keratin. Superficial mycoses probably represent the most prevalent infectious disease found worldwide. ${ }^{7}$ The fungus colonizes keratin tissues and causes inflammation as a result of the host's response to its metabolic by-products. The dermatophytes of Trichophyton rubrum are distributed over the world. ${ }^{8}$ T. rubrum is seldom isolated from animals and is rarely found in soil. The infections caused by dermatophytes may have different manifestations depending on the site of the infection. The inflammatory reaction caused by $T$. rubrum can cause severe damage to the skin and in particular, the nails. Recently, sequential pulse therapy with itraconazole (a triazole drug) and terbinafine (an allylamine drug) has been used to treat onmychomycosis of the fingernail. ${ }^{9-11}$ The objective of this study was to evaluate the antifungal activity of chalcones derived from $p$-aminoacetophenone and 3'-methoxy-4'hydroxyacetophenone against four strains of the dermatophytes of Trychophyton rubrum.

\section{Materials and Methods}

\subsection{Synthesis and characterization of chalcones}

The $p$-aminochalcone and $3^{\prime}$-methoxy-4'hydroxy chalcone derivatives used in this study were synthesized via Claisen-Schmidt condensation conducted under basic conditions. ${ }^{12} \mathrm{~A}$ solution of $p$-aminoacetophenone ( $2 \mathrm{mmol}$ ) and 3-methoxy-4hydroxyacetophenone $(2 \mathrm{mmol})$ in ethanol $(5 \mathrm{~mL})$ was added the to a solution of benzaldehyde (2 $\mathrm{mmol})$ in ethanol $(5 \mathrm{~mL})$ containing 10 drops of 50 $\% \mathrm{v} / \mathrm{v}$ sodium hydroxide, and the resulting mixture was stirred for $48 \mathrm{~h}$. The mixture was filtered under vacuum, washed with cold water to $\mathrm{pH} 7.0$, and analyzed by TLC (Figure 2 and 3 ).

The chemical reagents were from obtained Sigma-Aldrich. ${ }^{1} \mathrm{H}$ and ${ }^{13} \mathrm{C}$ NMR spectra were obtained using a Bruker Spectrometer, model Avance DPX - 300 and model Avance DRX-500 operating at a frequency of $300 \mathrm{MHz}$ and $500 \mathrm{MHz}$ for hydrogen, $75 \mathrm{MHz}$ and $125 \mathrm{MHz}$ for carbon respectively. The spectra were measured in $\mathrm{CD}_{3} \mathrm{OD}$, $\mathrm{CD}_{3} \mathrm{COCD}_{3}$ and $\mathrm{CDCl}_{3}$ solvents and chemical shifts are reported as $\delta$ values in parts per million (ppm) relative to tetramethylsilane $(\delta 0.00)$ as internal standard. The mass spectra were obtained with a Shimadzu QP201 GC-MS (Gas Chromatography coupled to Mass Spectrometry) using RTX-5MS capillary column $(30.0 \mathrm{~m} \times 0.25 \mathrm{~mm} \times 0.30 \mathrm{~mm})$ for compounds with in the literature record and UPLC-QTOF-MS performed in an ACQUITY UPLC BEH column ( $150 \times 2.1 \mathrm{~mm}, 1.7$ um; Waters Co.) on a Waters Acquity UPLC system. The column temperature was set at $40{ }^{\circ} \mathrm{C}$. The binary gradient

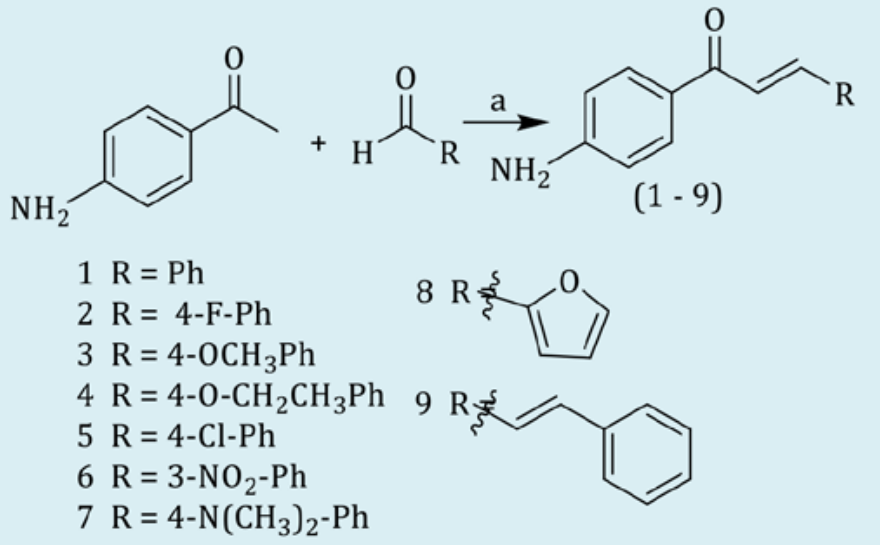

Figure 2. Preparation of chalcones (1 - 9) a) $\mathrm{NaOH} 50 \% \mathrm{w} / \mathrm{v}$, ethanol, r.t., $48 \mathrm{~h}$<smiles>[R]C=CC(=O)c1ccc(O)c(OC)c1</smiles>

Figure 3. Preparation of chalcone (10) a) $\mathrm{NaOH} 50 \% \mathrm{w} / \mathrm{v}$, ethanol, r.t., $48 \mathrm{~h}$ 
elution system consisted of $0.1 \%$ formic acid in water (A) and $0.1 \%$ formic acid in acetonitrile (B), with linear gradient from 2 to $95 \% \mathrm{~B}(0-15 \mathrm{~min})$, with a flow rate of $0.4 \mathrm{~mL}$. $\mathrm{min}^{-1}$ for the new compounds (6a) and (11). Infrared spectra were determined on a Perkin Elmer FT/IR 1000 spectrophotometer and are reported in wave number $\left(\mathrm{cm}^{-1}\right)$. The melting point was done in the apparatus MQAPF-302 (microchemistry) with heating rate $3.0^{\circ} \mathrm{C} / \mathrm{min}$.

\subsection{Fungal strains}

Experiments were conducted using T. rubrum LABMIC 0208, 0210, 0204, and 0209. The fungal strains were obtained from potato agar stock at $-20{ }^{\circ} \mathrm{C}$, gently yielded by the Laboratory of Microbiology of the Vale do Acaraú State University (UVA) and the Center Specialist in Medical Mycology (CEMM), Department of Pathology and Legal Medicine of the Federal University of Ceara. The fungal samples were peeled into tubes containing potato dextrose agar. (Difco, Detroit, $\mathrm{MI}$, USA) and incubated at $28^{\circ} \mathrm{C}$ for 10 days in order to verify the presence of colony growth. ${ }^{13,14}$

\subsection{Preparation of the inoculum used for the antifungal sensitivity tests}

After confirming the viability of the fungal strains, the cultures were covered with $5 \mathrm{~mL}$ of saline solution or sterile water with subsequent scraping of the surface of the colonies in order to obtain a suspension that was free of fragments in the culture medium. The solution containing conídia and hyphal filaments was diluted in a 1:5 proportion using RPMI 1640 medium with L-glutamine without sodium bicarbonate buffered at $\mathrm{pH} 7.0$ with (3-( $\mathrm{N}$-morpholino)propane sulfonic acid (MPOS; $0.165 \mathrm{M}$ solution), resulting in

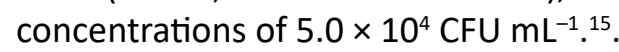

\subsection{Antifungal activity}

The minimum inhibitory concentration (MIC) and minimum fungicidal concentration (MFC) of the compounds against the dermatophytes were determined using the microdilution method in broth with 96-well plates according to CLSI M27A2. The compounds were prepared in (DMSO) at a concentration of $10 \mathrm{mg} \mathrm{mL}-1$, diluted in $100 \mu \mathrm{L}$ of RPMI 1640 medium (Sigma), and tested over a concentration range of $625-10000 \mu \mathrm{g} \mathrm{mL}-1$. Thereafter, $100 \mu \mathrm{L}$ of the inoculum was added to the 96-well plate. Ketoconazole, an antifungal drug, was used as a positive control in a concentration range of $0.07-16 \mu \mathrm{g} \mathrm{mL}-1$. The microplates were incubated at $37{ }^{\circ} \mathrm{C}$ and read visually after 5 days. The MIC was defined as the lowest concentration capable of inhibiting fungal growth. In turn, the minimum fungicidal concentration (MFC) was determined after transferring $100 \mathrm{~mL}$ of the contents of the well without turbidity into tubes containing potato agar at $28^{\circ} \mathrm{C}$. The MFC against the various dermatophytes was calculated according to the fungal growth in the culture medium after 5 days, whereas for the yeasts it was determined after $24 \mathrm{~h}$. Miroxylon peruiferum L. F. exhibits an MIC of $625 \mu \mathrm{g} \mathrm{mL}-1$ and CFM of $1250 \mu \mathrm{g} \mathrm{mL}-1$ for the two strains of T. rubrum, while Astronium fraxinifolium Schot exhibits a MIC of 2500 $\mu \mathrm{g} \mathrm{mL}-1$ and MFC of $5000 \mu \mathrm{g} \mathrm{mL}-1$ against the two strains tested.

\section{Results and Discussions}

\subsection{Analytical data}

(2E)-1-(4'-aminophenyl)-3-(phenyl)-prop-2en-1-one (1)

Yellow solid (Yield: $25.60 \%$ ), m.p. 109.3 109.9 $\mathrm{C}$; FT-IR $\left(\mathrm{KBr}, \mathrm{v}_{\mathrm{cm}}{ }^{-1}\right): 3522,3434,1623,1578$, 1554. ${ }^{1} \mathrm{H}-\mathrm{NMR}\left(\mathrm{CD}_{3} \mathrm{OD}, 300 \mathrm{MHz}\right) \delta: 7.40-7.42$ (m, H-3/H-5, H-4), 7.93 (d, H-2'/H-6', J = $8.7 \mathrm{~Hz}$ ), $6.73\left(\mathrm{~d}, \mathrm{H}-3^{\prime} / \mathrm{H}-5^{\prime}, J=8.7 \mathrm{~Hz}\right), 7.69-7.72(\mathrm{~m}$, $\mathrm{H}-2 / \mathrm{H}-6, \mathrm{H}-\alpha, \mathrm{H}-\beta) .{ }^{13} \mathrm{C}-\mathrm{NMR}\left(\mathrm{CD}_{3} \mathrm{OD}, 75 \mathrm{MHz}\right) \delta$ : C-1 136.8, C-2/C-6 129.6 C-3/C-5 130.1, C-4 131.4, C-1'128.2, C-2'/C-6' 132.6, C-3'/C-5' 115.1, C-4' 154.8, C- $\alpha$ 123.3, C- $\beta$ 144.4, C=O 190.3. MS (EI) $\mathrm{m} / \mathrm{z}\left(\mathrm{M}^{+} \cdot 223\right)$, calcd for $\mathrm{C}_{15} \mathrm{H}_{13} \mathrm{NO} / 223$.

(2E)-1-(4'-aminophenyl)-3-(4-fluophenyl)prop-2-en-1-one (2)

Yellow solid (Yield: $28 \%$ ), m.p. $161.5-162.5$ $9^{\circ} \mathrm{C}$; FT-IR $\left(\mathrm{KBr}, \mathrm{v}_{\mathrm{cm}}{ }^{-1}\right)$ : 3600, 1660, 1590, 1570. ${ }^{1} \mathrm{H}-\mathrm{NMR}\left(\mathrm{CD}_{3} \mathrm{OD}, 300 \mathrm{MHz}\right)$ 8: 7.76 (dd, H-2/H-6, $J=8.8 / 2.1 \mathrm{~Hz}), 7.14(\mathrm{t}, \mathrm{H}-3 / \mathrm{H}-5, J=8,8 \mathrm{~Hz}), 7.91$ (d, H-2'/H-6', J = $6.93 \mathrm{~Hz}), 6.73\left(\mathrm{~d}, \mathrm{H}-3^{\prime} / \mathrm{H}-5^{\prime}, J=\right.$ $8.8 \mathrm{~Hz}), 7.67(\mathrm{~d}, \mathrm{H}-\alpha, J=12.1 \mathrm{~Hz}), 7.74(\mathrm{~d}, \mathrm{H}-\beta, J=$ $14,2 \mathrm{~Hz}$ ). ${ }^{13} \mathrm{C}-\mathrm{NMR}\left(\mathrm{CD}_{3} \mathrm{OD}, 75 \mathrm{MHz}\right) \delta: \mathrm{C}-1$ 132.6, C-2/C/6 132.6, C-3/C-5 116.1, C-4 163.8, C-1' $128.1, \mathrm{C}-2^{\prime} / \mathrm{C}-6^{\prime} 131.8, \mathrm{C}-3^{\prime} / \mathrm{C}-5^{\prime}$ 114.9, C-4' 155.0, $\mathrm{C}-\alpha$ 123.2, C- $\beta$ 142.9, $\mathrm{C}=0$ 190.1. MS (EI) $\mathrm{m} / \mathrm{z}\left(\mathrm{M}^{+}\right.$. 241), calcd for $\mathrm{C}_{15} \mathrm{H}_{12} \mathrm{NOF} / 241$. 
(2E)-1-(4'-aminophenyl)-3-(4-methoxyphenyl)-prop-2-en-1-one (3)

Yellow solid (Yield: $38 \%$ ), m.p. $148-148,5^{\circ} \mathrm{C}$; FTIR (KBr, $\left.\mathrm{v}_{\mathrm{cm}}{ }^{-1}\right):$ 1628, 1570, $15601555,1480,1240$. ${ }^{1} \mathrm{H}-\mathrm{NMR}\left(\mathrm{CDCl}_{3}, 300 \mathrm{MHz}\right): \delta 7.92$ (d, H-2/H-6, J = $8.61 \mathrm{~Hz}), 6.70(\mathrm{~d}, \mathrm{H}-3 / \mathrm{H}-5, J=8.58 \mathrm{~Hz}), 7.59$ (d, $\left.\mathrm{H}-2^{\prime} / \mathrm{H}-6^{\prime}, J=8.64 \mathrm{~Hz}\right), 6.93\left(\mathrm{~d}, \mathrm{H}-3^{\prime} / \mathrm{H}-5^{\prime}, J=8.67\right.$ $\mathrm{Hz}), 7.42(\mathrm{~d}, \mathrm{H}-\alpha, J=15.57 \mathrm{~Hz}), 7,76(\mathrm{~d}, \mathrm{H}-\beta, J=15.39$ $\mathrm{Hz}), 3.89\left(\mathrm{~s}, \mathrm{OCH}_{3}\right) \cdot{ }^{13} \mathrm{C}-\mathrm{NMR}\left(\mathrm{CDCl}_{3}, 75 \mathrm{MHz}\right): \delta \mathrm{C}-1$ 128.3, C-2/C-6 131.1, C-3/C-5 114.2, C-4 161.5, C-1' 127.8, C-2'/C-6' 130.2, C-3'/C-5' 114.5, C-4' 151.1, $\mathrm{C}-\alpha$ 120.1, C- $\beta$ 143.2, $\mathrm{C}=\mathrm{O}$ 188.4, $\mathrm{OCH}_{3}$ 55.8). MS (EI) $\mathrm{m} / \mathrm{z}\left(\mathrm{M}^{+} \cdot 253\right)$, calcd for $\mathrm{C}_{16} \mathrm{H}_{15} \mathrm{NO}_{2} / 253$.

(2E)-1-(4'-aminophenyl)-3-(4-ethoxyphenyl)prop-2-en-1-one (4)

Yellow solid (Yield: $33.89 \%$ \%), m.p. 140 $140,8^{\circ} \mathrm{C}$; FT-IR $\left(\mathrm{KBr}, \mathrm{v}_{\mathrm{cm}}{ }^{-1}\right): 1634,1600,1588,1575$, 1480, 1167. ${ }^{1} \mathrm{H}-\mathrm{NMR}\left(\mathrm{CD}_{3} \mathrm{OD}, 300 \mathrm{MHz}\right): \delta 7.90(\mathrm{~d}$, $\mathrm{H}-2 / \mathrm{H}-6, J=8.70 \mathrm{~Hz}), 6.69(\mathrm{~d}, \mathrm{H}-3 / \mathrm{H}-5, J=8.73 \mathrm{~Hz}$ ), 7.63 (d, H-2'/H-6', J = 7,98 Hz), 6.94 (d, H-3'/H-5', J $=8.70 \mathrm{~Hz}), 7.58(\mathrm{~d}, \mathrm{H}-\alpha, J=15.57 \mathrm{~Hz}), 7.65(\mathrm{~d}, \mathrm{H}-\beta, J$ $=15.06 \mathrm{~Hz}), 4,08\left(\mathrm{q}, \mathrm{CH}_{2}, J=6.96 \mathrm{~Hz}\right), 1.39\left(\mathrm{t}, \mathrm{CH}_{3^{\prime}}\right.$ J $=6.99 \mathrm{~Hz}) \cdot{ }^{13} \mathrm{C}-\mathrm{NMR}\left(\mathrm{CD}_{3} \mathrm{OD}, 75 \mathrm{MHz}\right): \delta \mathrm{C}-1129.3$, C-2/C-6 132.5, C-3/C-5 114.6, C-4 155.5, C-1' 127.9, C-2'/C-6' 131.4, C-3'/C-5' 116.1, C-4' 162.6, C- $\alpha$ 120.7, $\mathrm{C}-\beta$ 144.4, $\mathrm{C}=\mathrm{O}$ 199.3, $\mathrm{CH}_{2}$ 64.8; $\mathrm{CH}_{3}$ 15.2. MS (EI) $\mathrm{m} / \mathrm{z}\left(\mathrm{M}^{+} \cdot 267\right)$, calcd for: $\mathrm{C}_{17} \mathrm{H}_{17} \mathrm{NO}_{2} / 267$.

(2E)-1-(4'-aminophenyl)-3-(4-clorophenyl)prop-2-en-1-one (5)

Yellow solid (Yield: 53.59 \%), m.p. 162,9 $163,3{ }^{\circ} \mathrm{C}$; FT-IR $\left(\mathrm{KBr}, \mathrm{vcm}^{-1}\right)$ : 3555, 3350, 1621, 1570, 1550, 1490. ${ }^{1} \mathrm{H}-\mathrm{NMR}\left(\mathrm{CD}_{3} \mathrm{OD}, 300 \mathrm{MHz}\right): \delta$ $7.91(\mathrm{~d}, \mathrm{H}-2 / \mathrm{H}-6, J=8.73 \mathrm{~Hz}), 7.69(\mathrm{~d}, \mathrm{H}-3 / \mathrm{H}-5$, $J$ $=8.79 \mathrm{~Hz}), 7.42\left(\mathrm{~d}, \mathrm{H}-2^{\prime} / \mathrm{H}-6^{\prime}, J=8.46 \mathrm{~Hz}\right), 6.68(\mathrm{~d}$, $\mathrm{H}-3^{\prime} / \mathrm{H}-5^{\prime}, J \mathrm{~Hz}$ ), $7.70(\mathrm{~d}, \mathrm{H}-\alpha, J=14.28 \mathrm{~Hz}), 7.74$ (d, $\mathrm{H}-\beta, J=15.60 \mathrm{~Hz}) \cdot{ }^{13} \mathrm{C}-\mathrm{NMR}\left(\mathrm{CD}_{3} \mathrm{OD}, 75 \mathrm{MHz}\right.$ ): $\delta$ C-1 135.6, C-2/C-6 132.7, C-3/C-5 130.9, C-4 137.5, C-1' 127.6, C-2'/C-6' 130.8, C-3'/C-5' 114.6, C-4' 155.8, C- $\alpha$ 124.3, C- $\beta$ 142.9, C=O 189.9. MS (EI) $\mathrm{m} / \mathrm{z}\left(\mathrm{M}^{+} \cdot 257.5\right)$, calcd for: $\mathrm{C}_{15} \mathrm{H}_{12} \mathrm{NOCl} / 257.5$.

(2E)-1-(4'-aminophenyl) -3- (3-nitrophenyl) prop-2-en-1-one (6)

Orange solid (Yield: $69.85 \%$ \%), m.p. 208 $208,7^{\circ} \mathrm{C}$; FT-IR $\left(\mathrm{KBr}, \mathrm{v}_{\mathrm{cm}}{ }^{-1}\right): 3400,1640,1600$, 980. ${ }^{1} \mathrm{H}-\mathrm{NMR}\left(\mathrm{CD}_{3} \mathrm{COCD}_{3}, 300 \mathrm{MHz}\right): \delta 8.26(\mathrm{~d}$, $\mathrm{H}-2, J=1.95 \mathrm{~Hz}), 7.98-8.26(\mathrm{~m}, \mathrm{H}-4 / \mathrm{H}-5), 7.75$ (d, H-2'/H-6, J = $7.89 \mathrm{~Hz}$ ), $6.74\left(\mathrm{~d}, \mathrm{H}-3^{\prime} / \mathrm{H}-5^{\prime}, J=\right.$ $8.67 \mathrm{~Hz}$ ), 7.72 (d, $\mathrm{H}-\alpha, J=15.96 \mathrm{~Hz}$ ), 7.80 (d, $\mathrm{H}-\beta$,
$J=17.34 \mathrm{~Hz}) \cdot{ }^{13} \mathrm{C}-\mathrm{NMR}\left(\mathrm{CD}_{3} \mathrm{COCD}_{3} 75 \mathrm{MHz}\right): \delta \mathrm{C}-1$ 138.6, C-2 124.9, C-3 149.9, C-4 126.3, C-5 131.2, C-6 135.2, C-1' 127.6, C-2'/C-6' 132.3 C-3'/C-5' 114.2, C-4' 154.7, C- $\alpha$ 123.9, C- $\beta$ 140.0, $C=0$ 186.9. MS (EI) $\mathrm{m} / \mathrm{z}\left(\mathrm{M}^{+} \cdot 268\right)$, calcd for $\mathrm{C}_{15} \mathrm{H}_{12} \mathrm{~N}_{2} \mathrm{O}_{3} / 268$.

( $2 E)-1-\left(4^{\prime}-a m\right.$ i noph e n y I) -3-(4-dimethyaminophenyl) -prop-2-en-1-one (7) Orange solid (Yield: $35.66 \%$ ), m.p. $169-170^{\circ} \mathrm{C}$; $\mathrm{FT}-\mathrm{IR}\left(\mathrm{KBr}, \mathrm{v}_{\mathrm{cm}}{ }^{-1}\right): 3500,3460,1610,1570,1350,1160$, 980. ${ }^{1} \mathrm{H}-\mathrm{NMR}\left(\mathrm{CD}_{3} \mathrm{OD}, 300 \mathrm{MHz}\right): \delta 7.57$ (d, H-2/H-6, $J=8.85 \mathrm{~Hz}), 6.69(\mathrm{~d}, \mathrm{H}-3 / \mathrm{H}-5, J=8.76 \mathrm{~Hz}), 7.89(\mathrm{~d}$, $\left.\mathrm{H}-2^{\prime} / \mathrm{H}-6^{\prime}, J=8.73 \mathrm{~Hz}\right), 6.76\left(\mathrm{~d}, \mathrm{H}-3^{\prime} / \mathrm{H}-5^{\prime}, J=8.88 \mathrm{~Hz}\right)$, $7.46(\mathrm{~d}, \mathrm{H}-\alpha, J=15.39 \mathrm{~Hz}), 7.68(\mathrm{~d}, \mathrm{H}-\beta, J=15.39$ $\mathrm{Hz}$ ), $3.02\left(\mathrm{~s}, 2 \mathrm{CH}_{3}\right) \cdot{ }^{13} \mathrm{C}-\mathrm{NMR}\left(\mathrm{CD}_{3} \mathrm{OD}, 75 \mathrm{MHz}\right): \delta \mathrm{C}-1$ 124.7, C-2/C-6 131.5, C-3/C-5 113.3, C-4 153.8, 2CH 40.4, C-1' 128.4, C-2'/C-6' 132.3, C-3'/C-5' 114.6, C-4' 155.2, C- $\alpha$ 117.6, C- $\beta$ 145.8, C=O 190.8. MS (EI) $\mathrm{m} / \mathrm{z}\left(\mathrm{M}^{+} \cdot 266\right)$, calcd for $\mathrm{C}_{17} \mathrm{H}_{18} \mathrm{~N}_{2} \mathrm{O} / 266$.

(2E)-1-(4'-aminophenyl) -3- furan-2-yl-prop-2en-1-one (8)

Dark orange solid (Yield: 69,85 \%), m.p. 118 $118,3^{\circ} \mathrm{C}$; FT-IR $\left(\mathrm{KBr}, \mathrm{v}_{\mathrm{cm}}{ }^{-1}\right): 3447,3434,1640,1584$, 1545, 1173. ${ }^{1} \mathrm{H}-\mathrm{NMR}\left(\mathrm{CD}_{3} \mathrm{OD}, 300 \mathrm{MHz}\right): \delta 6.78$ (d, $\mathrm{H}-3, \mathrm{~J}=3.36 \mathrm{~Hz}), 6.55$ (m, H-4); $7.62(\mathrm{~s}, \mathrm{H}-5), 7,85$ (d, $\left.\mathrm{H}-2^{\prime} / \mathrm{H}-6^{\prime}, J=8.73 \mathrm{~Hz}\right), 6.68\left(\mathrm{~d}, \mathrm{H}-3^{\prime} / \mathrm{H}-5^{\prime}, J=8.73 \mathrm{~Hz}\right)$, $7.50-7.62$ (s broad, $\mathrm{H}-\alpha, \mathrm{H}-\beta) .{ }^{13} \mathrm{C}-\mathrm{NMR}\left(\mathrm{CD}_{3} \mathrm{OD}, 75\right.$ MHz): $\delta$ C-2 153.4, C-3 113.7, C-4 116.5, C-5 146.3, C-1 127.6, C-2`/C-6`132.4, C-3`/C-5`114.6, C-4`155.5, C-2 153.4, C-3 113.7, C- $\alpha$ 130.5, C- $\beta 120.5, C=0$ 189.7. MS (EI) $\mathrm{m} / \mathrm{z}\left(\mathrm{M}^{+}\right.$213), calcd for $\mathrm{C}_{13} \mathrm{H}_{11} \mathrm{NO}_{2} / 213$.

(2E,4E)-1-(4-aminophenyl)-5-phenylpenta-

\section{2,4-dien-1-one (9)}

Dark orange solid (Yield: 34 \%), m.p. 151,8 $152^{\circ} \mathrm{C}$; FT-IR (KBr, $\left.\mathrm{v}_{\mathrm{cm}}{ }^{-1}\right): 3458,3376,1635,1611$, 1576 1564. ${ }^{1} \mathrm{H}-\mathrm{NMR}\left(\mathrm{CD}_{3} \mathrm{OD}, 300 \mathrm{MHz}\right): \delta 7.26-$ $7.35(\mathrm{~m}, \mathrm{Ar}), 7.84\left(\mathrm{~d}, \mathrm{H}-2^{\prime} / \mathrm{H}-6^{\prime}, J=8.79 \mathrm{~Hz}\right) .6 .67$ (d, $\mathrm{H}-3^{\prime} / \mathrm{H}-5^{\prime}, J=8.76 \mathrm{~Hz}$ ). $7.00-7.25$ (m, H- $\alpha$, $\mathrm{H}-7, \mathrm{H}-8), 7.38$ (ddd, $\mathrm{H}-\beta, J=15.96,8.43,1.65 \mathrm{~Hz}$ ). ${ }^{13} \mathrm{C}-\mathrm{NMR}\left(\mathrm{CD}_{3} \mathrm{OD}, 75 \mathrm{MHz}\right): \delta \mathrm{C}-1$ 138.0, C-2/C-6 128.7, C-3/C-5 130.0, C-4 128.4, C-7 126.8, C-8 142.6, C-1 $127.7, \quad C-2^{\prime} / C-6^{\prime} 132.5, \quad C-3^{\prime} / C^{\prime}-5^{\prime}$ 114.6, $C-4155.6, C-\alpha$ 130.5, C- $\beta$ 144.6, $C=0$ 190.3. $\mathrm{MS}$ (EI) $\mathrm{m} / \mathrm{z}\left(\mathrm{M}^{+} \cdot 253\right)$, calcd for $\mathrm{C}_{17} \mathrm{H}_{15} \mathrm{NO} / 253$.

(2E) -1- (3'-methoxy-4'-hydroxyphenyl) -3(3-nitrophenyl) prop-2-en-1-one (10)

Yellow solid (Yield: 46,33\%), m.p. $179.4-180^{\circ} \mathrm{C}$; FT-IR $\left(\mathrm{KBr}, \mathrm{v}_{\mathrm{cm}}{ }^{-1}\right)$ : 3425, 1621, 1575, 1350, 990. ${ }^{1} \mathrm{H}-\mathrm{NMR}\left(\mathrm{CD}_{3} \mathrm{COCD}_{3}, 300 \mathrm{MHz}\right): \delta 6.96-8.26(\mathrm{~m}, \mathrm{Ar})$, 
8.65 (s, H-2'), $7.73\left(\mathrm{~m}, \mathrm{H}-5^{\prime}\right), 8.23\left(\mathrm{~d}, \mathrm{H}-6^{\prime}, J=6.36\right.$ $\mathrm{Hz}$ ), 7.82 (d, $\mathrm{H}-\alpha, J=15.35 \mathrm{~Hz}$ ), $7.87(\mathrm{~d}, \mathrm{H}-\beta, J=15.35$ $\mathrm{Hz}), 3.90\left(\mathrm{~s}, \mathrm{OCH}_{3}\right){ }^{13} \mathrm{C}-\mathrm{NMR}\left(\mathrm{CD}_{3} \mathrm{COCD}_{3} 75 \mathrm{MHz}\right)$ : $\delta$ C-1 131.1, C-2 124.9, C-3 152.9, C-4 125.1, C-5 131.2, C-6 135,4, C-1`138.3, C-2`124.9, C-3`148.8, C-4`149.9, C-5`115.6, C-6`125.8, C- $\alpha 123.5, C-\beta$ 141.1, $\mathrm{OCH}_{3}$ 56.5, $\mathrm{C}=\mathrm{O}$ 187.8. HRESIMS, $\mathrm{m} / \mathrm{z}$ : $300.0867\left(\mathrm{C}_{16} \mathrm{H}_{13} \mathrm{NO}_{3}\right)[\mathrm{M}+\mathrm{H}]^{+}$(calcd. 300.0872).

\subsection{Chemistry}

The synthesis of chalcones in this work was carried out by a Claisen-Schmith condensation reaction inbasicmedium ${ }^{11}$. Accordingtotheproduct formed, the base acting as a catalyst removes an $\alpha$ hydrogen from the $p$-aminoacetophenone, transforming it into a nucleophile, which then reacts with the carbonyl carbon of the aldehyde that acts as an electrophile. The reaction precedes the release of a water molecule and formation of the chalcone with the $\alpha, \beta$-unsaturated conjugate system. The reaction yield ranges from $25.60-$ $69.85 \%$. The variation in yield is dependent on the nature of the group attached to ring $B$. The presence of electron-withdrawing groups leads to greater yield. The presence of electron removal groups in the ring produces a lower yield.

The structures of the $p$-aminochalcone and 3'-methoxy-4'-hydroxy chalcone derivatives were determined using nuclear magnetic resonance (NMR) and infra-red (IR) spectroscopies, and mass spectrometry (MS). The ${ }^{1} \mathrm{H}$ NMR spectra indicate that only the $E$-isomers were formed in the ClaisenSchmidt condensation reaction. In the ${ }^{1} \mathrm{H}$ NMR spectra obtained for the chalcone derivatives, the signal observed at $\delta=4.81 \mathrm{ppm}$ corresponds to the hydrogen atoms in the amino group. The doublets observed at $\delta=7.75$ and $7.94 \mathrm{ppm}(\mathrm{J}=15.6 \mathrm{~Hz})$ were attributed to the $\alpha, \beta$-unsaturated hydrogen atoms, whose coupling constants $(\mathrm{J})$ confirmed the $E$ geometry of the double bond. For ring $B$ in the chalcone derivatives, three signals were observed between $\delta=7.42-7.60$, a doublet of doublets corresponding to the hydrogen atoms at $\mathrm{C} 2 / \mathrm{C} 6$, and two multiplets attributed to the hydrogen atoms at $\mathrm{C} 3 / \mathrm{C} 5$ and $\mathrm{C} 4$. For ring $\mathrm{B}$ in chalcones $\mathbf{2}$ and $\mathbf{3}$, two doublets were observed between $\delta=7.38$ $7.65 \mathrm{ppm}$ corresponding to the hydrogen atoms at $\mathrm{C} 2 / \mathrm{C} 6$ and $\mathrm{C} 3 / \mathrm{C} 5$. For ring $\mathrm{B}$ in chalcone 4 , four signals were observed at $\delta=8.45$ corresponding to the hydrogen atom at $\mathrm{C} 2$, a doublet at $\delta=7.59$ attributed to the hydrogen atom at $\mathrm{C} 5$, and two doublet peaks at $\delta=8.22(\mathrm{~d}, \mathrm{~J}=8,13 \mathrm{~Hz})$ and 7.86 (d, $J=7.60 \mathrm{~Hz}$ ) assigned to the hydrogens atoms at $\mathrm{C} 4$ and $\mathrm{C} 6$, respectively. In the ${ }^{13} \mathrm{C}$ NMR spectra obtained for the synthesized chalcone derivatives, it was possible to observe the peak corresponding to the $\alpha, \beta$-unsaturated carbonyl atom for $\delta=192.7-$ $193.5 \mathrm{ppm}$. The olefinic $\alpha$ and $\beta$ carbon atoms were observed at $\delta=127.4$ and $142.8 \mathrm{ppm}$, respectively.

The infrared data corroborated the confirmation of the structure, affirming the presence of stretch bands characteristic of $C=O$ with values of system conjugated to C-sp2 and stretch bands of the trans type demonstrating the formation of the double bond $C=C$. Stretch bands $C=C$, characteristic of mono, meta and substituted aromatic rings, are also observed. These and other values are found in tables 1 and 2.

The structures of the synthesized and

Table 1. Infrared data for 4'-aminochalcones (1-9)

\begin{tabular}{|c|c|c|c|c|c|c|c|c|c|}
\hline \multirow{2}{*}{$\begin{array}{l}\text { Main } \\
\text { vibrations }\end{array}$} & \multicolumn{9}{|c|}{ Chalcones } \\
\hline & 1 & 2 & 3 & 4 & 5 & 6 & 7 & 8 & 9 \\
\hline v N-H & $3452 / 3415$ & $3460 / 3340$ & $3450 / 3400$ & - & $3455-3345$ & $3400 / 3380$ & $3500 / 3460$ & $3425 / 3390$ & $3460 / 3380$ \\
\hline$\vee C=O$ & 1662 & 1630 & 1630 & 1660 & 1622 & 1640 & 1610 & 1630 & 1615 \\
\hline$\vee C=C$ & $1600-1420$ & $1600-1550$ & $1600-1500$ & $1600-1520$ & $1660-1478$ & $1600-1540$ & $1570-1500$ & $160-1470$ & $1600-1530$ \\
\hline v C-N & 1334 & 1340 & 1310 & 1320 & 1345 & 1330 & $1350 / 1310$ & 1315 & 1340 \\
\hline v C-O & - & - & 1180 & 1170 & 1180 & - & 1160 & 1180 & - \\
\hline$\delta \mathrm{C}-\mathrm{H}_{\text {Trans }}$ & 970 & 970 & 975 & 960 & 975 & 980 & 950 & 980 & 985 \\
\hline$\delta \mathrm{C}-\mathrm{H}_{\text {Mono }}$ & $790 / 750$ & - & - & - & - & - & - & - & - \\
\hline$\delta \mathrm{C}-\mathrm{H}_{\text {Meta }}$ & - & - & - & - & - & $680 / 650 / 590$ & - & - & - \\
\hline$\delta \mathrm{C}-\mathrm{H}_{\text {Para }}$ & 820 & 820 & 820 & 850 & 820 & 840 & 820 & 810 & 815 \\
\hline$\delta C(C=O) C$ & 1220 & 1220 & 1230 & 1260 & 1250 & 1220 & 1230 & 1225 & 1250 \\
\hline$\delta C-F$ & - & 1140 & - & - & - & - & - & - & - \\
\hline$\delta \mathrm{C}-\mathrm{Cl}$ & - & - & - & - & 1088 & - & - & - & - \\
\hline
\end{tabular}


Table 2. Infrared data for (2E)-1- (3'-methoxy-4'-hydroxyphenyl) -3- (3-nitrophenyl) prop-2-en-1-one (10)

\begin{tabular}{|c|c|}
\hline \multirow{2}{*}{ Main vibrations } & Chalcone \\
\hline & 10 \\
\hline v O-H & 3425 \\
\hline$v \mathrm{C}=\mathrm{O}$ & 1621 \\
\hline$\vee C=C$ & $1575-1465$ \\
\hline$v \mathrm{C}-\mathrm{N}$ & 1350 \\
\hline v C-O & 1160 \\
\hline$\delta \mathrm{C}-\mathrm{H}_{\text {Trans }}$ & 990 \\
\hline$\delta \mathrm{C}-\mathrm{H}_{\text {Mono }}$ & - \\
\hline$\delta \mathrm{C}-\mathrm{H}_{\text {Meta }}$ & $785 / 750 / 660$ \\
\hline$\delta \mathrm{C}-\mathrm{H}_{\text {Para }}$ & - \\
\hline$\delta C(C=O) C$ & 1190 \\
\hline$\delta \mathrm{C}-\mathrm{Cl}$ & - \\
\hline
\end{tabular}

acetylated chalcones were also confirmed through the analysis of the mass spectra in a fragmentation proposal, whose ions formed come from an a segmentation, which from there, there is the loss of a $\mathrm{CO}$ molecule by inductive segmentation, generating a second fragment ion. The mass spectra revealed peaks of the $\mathrm{M}^{+}$ molecular ion. Justifying the molecular formulas of the synthesized and acetylated chalcones, in addition to the base peak characteristic of the general process of fragmentation of chalcones. The data were compared with the literature of chalcones of similar structures. (Figures 4 and 5).

\subsection{Antifungal activity}

The microdilution assays conducted against the dermatophytes using the chalcone derivatives are summarized in Table 3 and show MIC values ranging from 0.015 to $1.25 \mathrm{\mu g} \mathrm{mL}^{-1}$ against $T$. rubrum LABMIC 0208, 0210, 0204, and 0209. However, (2E)- 1-(4'-aminophenyl)-3-(phenyl)-prop-2-en-1-one (1), (2E)-1-(4'-aminophenyl)-3-(4-fluorophenyl)prop-2-en-1-one (2), (2E)-1-(4'-aminophenyl)-3(4-chlorophenyl)-prop-2-en-1-one (5), (2E)-1-(4'aminophenyl)-3- furan-2-yl-prop-2-en-1-one (8), and (2E)-1-(3'-methoxy-4'-hydroxyphenyl)-3-(3nitrophenyl) prop-2-en-1-one (10) show activity against $T$. rubrum LABMIC 0208 (MIC $=0.07 \mu \mathrm{g}$ $\left.\mathrm{mL}^{-1}\right)$ and LABMIC $0204\left(\mathrm{MIC}=0.07 \mu \mathrm{g} \mathrm{mL}^{-1}\right)$.

The results revealed that $p$-aminochalcone derivatives bearing a phenyl group and electronwithdrawing substituents, such as fluorine and chlorine, as well as the presence of a heterocyclic ring show enhanced activity when compared to those with electron-donating substituents. In addition, the presence of the hydroxy and methoxy groups in ring A of 3'-methoxy-4'hydroxy chalcone as well as a nitro group on ring B appears to be important for activity against the dermatophytes studied.<smiles>COc1cc(C(=O)C=Cc2cccc([N+](=O)[O-])c2)ccc1O</smiles>

$\mathrm{m} / \mathrm{z} 299$<smiles>COc1cc(C#[Fe+])ccc1O</smiles>

$\mathrm{m} / \mathrm{z} 151$<smiles>COc1ccccc1O</smiles>

$\mathrm{m} / \mathrm{z} 123$

Figure 4. General fragmentation mechanism justifying the fundamental peaks of 3'-methoxy-4'-hydroxy chalcone (10) 
<smiles>[R]c1ccc(/C=C/C(=O)c2ccc(N)cc2)cc1</smiles>

$\mathrm{R}=\mathrm{H} \quad \mathrm{m} / \mathrm{z} 223$

$\mathrm{R}=\mathrm{F} \quad \mathrm{m} / \mathrm{z} 241$

$\mathrm{R}=\mathrm{OCH} 3 \mathrm{~m} / \mathrm{z} 253$

$\mathrm{R}=\mathrm{OCH}_{2} \mathrm{CH}_{3} \mathrm{~m} / \mathrm{z} 267$

$\mathrm{R}=\mathrm{Cl} \mathrm{m} / \mathrm{z} \quad 257,5$

$\mathrm{R}=\mathrm{NO}_{2} \quad \mathrm{~m} / \mathrm{z} 268$

$\mathrm{R}=\mathrm{N}\left(\mathrm{CH}_{3}\right)_{2} \quad \mathrm{~m} / \mathrm{z} 266$<smiles>[R]C(=O)c1ccc(N)cc1</smiles>

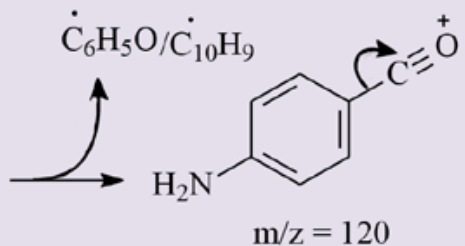<smiles>CC1CC1CC(=O)[O-]</smiles><smiles>[R]=C=Cc1ccco1</smiles>
$\mathrm{m} / \mathrm{z} 213$<smiles>[R10][R16]([H])([H])[H]</smiles>

Figure 5. General fragmentation mechanism justifying the fundamental peaks of 4'-aminochalcones (1-9)

Table 3. In vitro inhibitory concentration of chalcones

\begin{tabular}{|c|c|c|c|c|c|c|c|c|}
\hline \multicolumn{9}{|c|}{ Fungal strains } \\
\hline \multirow[t]{2}{*}{ Chalcones } & \multicolumn{2}{|c|}{$\begin{array}{c}\text { T. rubrum } \\
\text { LAMBIC } 0208\end{array}$} & \multicolumn{2}{|c|}{$\begin{array}{l}\text { T. rubrum } \\
\text { LAMBIC } 0210\end{array}$} & \multicolumn{2}{|c|}{$\begin{array}{c}\text { T. rubrum } \\
\text { LAMBIC } 0204\end{array}$} & \multicolumn{2}{|c|}{$\begin{array}{c}\text { T. rubrum } \\
\text { LAMBIC } 0209\end{array}$} \\
\hline & CIM & CFM & CIM & CFM & CIM & CFM & CIM & CFM \\
\hline 1 & 0.07 & 0.015 & 0.03 & 0.07 & 0.07 & 0.15 & 0.15 & 0.31 \\
\hline 2 & 0.15 & 0.31 & 0.07 & 0.015 & 0.07 & 0.15 & 0.015 & 0.031 \\
\hline 3 & 0.31 & 0.62 & 0.62 & 1.25 & 0.31 & 0.62 & 0.15 & 0.31 \\
\hline 4 & 0.31 & 0.62 & 0.31 & 0.62 & 0.15 & 0.31 & 0.31 & 0.62 \\
\hline 5 & 0.07 & 0.15 & 0.07 & 0.15 & 0.15 & 0.31 & 0.07 & 0.15 \\
\hline 6 & 0.62 & 1.25 & 0.62 & 1.25 & 0.62 & 1.25 & 0.62 & 1.25 \\
\hline 7 & 0.62 & 1.25 & 0.62 & 1.25 & 0.62 & 1.25 & 0.62 & 1.25 \\
\hline 8 & 0.07 & 0.15 & 0.07 & 0.15 & 0.31 & 0.62 & 0.07 & 0.15 \\
\hline 9 & 0.31 & 0.62 & 0.31 & 0.62 & 0.62 & 1.25 & 0.15 & 0.30 \\
\hline 10 & 0.07 & 0.15 & 0.07 & 0.15 & 0.07 & 0.15 & 0.15 & 0.31 \\
\hline
\end{tabular}

CIM and CFM $(\mu \mathrm{g} / \mathrm{mL})$ 


\section{Conclusion}

A series of ten chalcones was synthesized via the Claisen-Schmidt condensation reaction including nine 4'-aminochalcone derivatives and 3'-methoxy-4'-hydroxychalcone. A microdilution assay against the dermatophytes of $T$. rubrum showed that the $p$-aminochalcone derivatives bearing a phenyl group and electron-withdrawing substituents such as fluorine and chlorine atoms as well as the presence of a heterocyclic ring, have been shown to be more active than those with electron-donating substituents. In addition, the presence of the hydroxy and methoxy groups on ring $A$ in 3'-methoxy-4'-hydroxy chalcone as well as a nitro group on ring $B$ appears to be important for activity against the dermatophytes studied.

\section{Acknowledgments}

We would like to thank Northeastern Center for the Use and Application of Nuclear Magnetic Resonance (CENAUREM) for the spectra performed, and Editage for English language editing.

\section{References}

${ }^{1}$ Abbas, A.; Naseer, M. M.; Hasan, A.; Hadda, T. B. Synthesis and Cytotoxicity of 4-Alkoxychalcones as New Antitumor Agents. Journal Materials \& Environmental Science 2014, 5, 281. [Link]

2 Di Carlo, G.; Mascolo. N.; Izzo, A. A.; Capasso, F. Flavonoids: Old and New aspects of a class of natural therapeutic drugs. Life Science. 1999, 65, 337. [CrossRef] [PubMed]

${ }^{3}$ Boeck, P.; Leal, P. C.; Yunes, R. A.; Filho, V. C.; Lopez, S.; Sortino, M.; Escalante, A.; Furlan, R. L. E.; Zacchino S. Antifungal Activity and Studies on Mode of Action of Novel Xanthoxiline-drived Chalcones. Archiv der Pharmazie 2005, 338, 87. [CrossRef] [PubMed]

${ }^{4}$ Lopez, S. N.; Castelli, M. V.; Zacchino, S. A.; Dominguez, J. N.; Lobo, C.; Charris-Charris, J.; Cortes, J. C. G.; Ribas, J. C.; Devia, C.; Rodriguez, A. M.; Entriz, R. D. In Vitro Antifungal Evaluation and Structure-Activity Relationships of a New Series of Chalcone Derivatives and Synthetic Analogues, with Inhibitory Properties Against Polymers of the Fungal Cell Wall. Bioorganic Medicinal Chemistry 2001, 9, 1999. [CrossRef] [PubMed]
${ }^{5}$ Asif, M. A review on recent Advances and potential pharmacological activities of versatile chalcone molecule. Chemistry International 2016, 2, 1. [Link] ${ }^{6}$ Bag, S.; Ramar, S.; Degani, M. S. Synthesis and biological evaluation of $\alpha, \beta$-unsaturated ketone as potential antifungal agents. Medicinal Chemistry Research 2009, 18, 309. [CrossRef]

${ }^{7}$ Smijs, T. G. M.; Schuitmaker, H. J. Photodynamic Inactivation of the Dermatophyte Trichophyton rubrum. Phytochemistry and Photobiology 2003, 77, 556. [CrossRef]

${ }^{8}$ Aly, R. Ecology and epidemiology of dermatophyte infections. Journal American Academy Dermatology 1994, 31, 21. [CrossRef]

${ }^{9}$ Hart, R.; Bell-Syer, S. E. M.; Crawford, F.; Torgerson, D. J.; Young, P.; Russel, I. Systematic review of topical treatments for fungal infections of the skin and nails of the feet. Br. Medical Journal 1999, 19, 79. [CrossRef] [PubMed]

${ }^{10}$ Tosti, A.; Piraccini, B. M.; Stinchi, C.; Venturo, N.; Bardazzi, F. M. Colombo, M. D. Treatment of dermatophyte nail infections: an open randomised study comparing intermittent terbinafine treatment and intermittent itraconazoletherapy.Journal American Academy Dermatology 1996, 34, 595. [CrossRef]

${ }^{11}$ Faergemann, J.; Gupta, A. K.; Al Mofadi, A.; Abanami, A.; Shareaah, A. A, Marynissen, G. Efficacy of itraconazole in the prophylactic treatment of pityriasis (tinea) versicolor. archives of dermatology 2002, 138, 69. [CrossRef] [PubMed]

${ }^{12}$ Bhat, B. A.; Dhar, K. L.; Puri, S. C.; Saxena, A.K.; Shanmugavel, M.; Qazi, G. N. Synthesis and biological evolution of chalcones and their derived pyrazoles as potential cytotoxic agents. Bioorganic Medicinal Chemistry Letters 2005, 15, 3177. [CrossRef]

${ }^{13}$ Fontenelle, R.O.S.; Morais, S.M.B.; Mendonça, E.H.S.; Mendonça, M.R.; Brilhante K.; Cordeiro, R. A.; Nascimento, N. R. F.; Sidrim, J. J. C.; Rocha, M. F. G. Chemical composition, toxicological aspects and antifungal activity of essential oil from Lippia sidoides Cham. Journal of Antimicrobial Chemotherapy 2007, 59, 934. [CrossRef] [PubMed] ${ }^{14}$ Fontenelle, R. O. S.; Morais, S. M.; Brito. E. H. S.; Brilhante, R. S. N.; Cordeiro, R. A.; Nascimento, N. R. F.; Kerntopf, M. R.; Sidrim, J. J. C.; Rocha, M. F. G. Antifungal activity of essential oils of Croton species from the Brazilian Caatinga biome. Journal Applied Microbiology 2008, 104, 1383. [CrossRef] [PubMed] 15 Mosmann, T. Rapid Colorimetric Assay for Cellular Growth and Surviral: Application to Proliferation and Cytotoxicity Assays. Journal Immunology Methods 1983, 65, 55. [PubMed] 